\title{
PARASITOS DE BUFO SPINULOSUS WEIGMANN, 1835 Y TELMATOBIUS MARMORATUS PSEUDOJELSKII WEIGMANN, 1834 DE LA GRANJA K’AYRA,CUSCO.
}

ANTONIETA RECHARTE C.

Facultad de Ciencias Biológicas, Universidad San Antonio de Abad del Cusco, Cusco, Perú.

\begin{abstract}
RESUNEN
Se mencionan los parásitos protozoos, helmintos e hirudineos de Bufo spinulosus y Telmatobius marmoratus pseudojelskii de la granja $\mathrm{K}$ 'ayra del Cusco. Se establece que T. m. pseudojelskii es un nuevo húesped intermediario para Cylindrotaenia americana y Aplectana hylambatis. También se establece la relación entre el peso y longitud del huésped y sus parásitos.

PALABRAS CLAVE: Protozooa. Helmintos, Hirudinea. Cusco, Perú.
\end{abstract}

\section{SUMMARY}

In this paper, protozoa, helmints and hirudinea are recorded of Bufo spinulosus and Telmatobius marmoratus pseudojelskii from granja $\mathrm{K}$ 'ayra, Cusco, Perú. T. m. pseudojelskii is a new host for Cylindrotaenia americana and Aplectana hylambatis. The relation of the parasites and the lengt and weigth of the host are established.

KEY WORDS : Protozoa. Helmints. Hirudinea. Cusco, Peru.

\section{INTRODUCCCION}

Bufo spinolosus y Telmatobius marmoratus pseudojelskii son anfibios de importancia económica para el país y en especial para la región Inca; es así que B.spinolosus constituye un excelente controlador biológico de insectos, además de servir de material para estudios parasitológicos, anatómicos y fisiológicos, tanto en biología como en medicina. T.m.pseudojelskii, constituye una fuente proteica en la alimentación del hombre,especialmente en la sierra y la selva. El estudio de los parásitos de los animales silvestres es de gran interés, porque es posible encontrar especies propias de la región y también porque en muchos casos, los animales silvestres son reservorios de parásitos que atacan al hombre y a los animales domésticos.

En el presente trabajo, se identifican a las especies parásitas y se establece su prevalencia tanto en Bufo spinulosus como de Telmatobius marmoratus pseudojelskii de la granja K' ayra del Cusco y se estudia la relación existente entre los parasitos hallados y el peso y talla de sus huéspedes.

\section{MATERIAL Y METODOS.}

Se estudiaron 50 especímenes de Bufo spinulosus Wiegmann, 1835 y 50 de Telmatobius marmoratus pseudojelskii Wiegmann, 1834, Los especímenes de Bufo se colectaron en un área de $1364 \mathrm{~m}^{2}$ a una altitud de 3219 msnm. denominado potrero $2 \mathrm{Ht}$ de la estación Experimental del Centro Agronómico de K'ayra, que se encuentra a cargo de la Facultad de Agronomía y Zootecnia de la Universidad Nacional San Antonio de Abad del Cusco. Los Telmatobius se colectaron del riachuelo Huanacaure, ubicado en la parte Nor-Este de la granja mancionada. Todos los huéspedes fueron autopsiados en el laboratorio, sus órganos separados y abiertos, ubicando y separando los parásitos para luego lavarlos en solución salina fisiológica y fijarlos en alcohol-formol acético a $56^{\circ} \mathrm{C}$ o en solución MIF.

Para la identificación, los céstodos se colorearon con Carmín acético de Semichon y los nemátodos se diafanizaron en una mezcla de alcohol-fenol. Los hirudineos se identificaron previa fijación. Los protozoos se estudiaron in vivo y fotografiados; sus características se compararon con las descritas en publicaciones especializadas, como las de Amaro (1971).

La relación entre el peso y la talla del huésped con la carga parasitaria se estableció utilizando regresión lineal simple, por el método de los mínimos cuadrados para $\mathrm{X} \mathrm{e}$ $\mathrm{Y}$, tomando en cuenta un total de 50 ejemplares para especies.

\section{RESULTADOS}


Tabla 1. Prevalencia parasitaria de 50 Bufo spinulosus $y$ 50 Telmatobius marmoratus pseudojelskii de la granja K'ayra,Cusco.

\begin{tabular}{|lccccc|}
\hline Huésped & \multicolumn{2}{c}{ Parasitados } & \multicolumn{3}{c|}{ No Parasitados } \\
\cline { 2 - 5 } & $\mathrm{N}^{\circ}$ & $\%$ & & $\mathrm{~N}^{\circ}$ & $\%$ \\
\hline B. spinulosus & 47 & 94 & 3 & 6 \\
\hline T.m. pseudojelskii & 47 & 94 & 3 & 6 \\
\hline
\end{tabular}

Tabla 2.- Frecuencia relativa por especies parásitas en Bufo spinulosus de la granja K'ayra, Cusco.

\begin{tabular}{|c|c|c|c|c|c|c|}
\hline \multirow{2}{*}{} & \multicolumn{2}{|c|}{ Protozoarios } & \multicolumn{3}{c|}{ Nemátodos } & Céstodo \\
\cline { 2 - 7 } Hospedador & $\begin{array}{c}\text { Zelleriella } \\
\text { s.p }\end{array}$ & $\begin{array}{c}\text { Nyctotherus } \\
\text { cordiformis }\end{array}$ & $\begin{array}{c}\text { Hedruris } \\
\text { moniezi }\end{array}$ & $\begin{array}{c}\text { Aplectana } \\
\text { hylambatis }\end{array}$ & $\begin{array}{c}\text { Falcaustra } \\
\text { mascula }\end{array}$ & $\begin{array}{c}\text { Cylindrotaenia } \\
\text { americana }\end{array}$ \\
\hline Positivo & 16 & 4 & 2 & 27 & 4 & 31 \\
\hline$\%$ & 32 & 8 & 4 & 54 & 8 & 62 \\
\hline Negativo & 34 & 46 & 48 & 23 & 46 & 19 \\
\hline$\%$ & 68 & 92 & 96 & 46 & 92 & 38 \\
\hline
\end{tabular}

Tabla 3.- Frecuencia relativa por especies parásitas en Telmabịus marmoratus pseudojelskii de la granja k’ayra, Cusco.

\begin{tabular}{|c|c|c|c|c|c|c|c|}
\hline \multirow{2}{*}{$\begin{array}{l}\text { Hospe- } \\
\text { dador }\end{array}$} & \multicolumn{2}{|c|}{ Protozoarios } & \multicolumn{3}{|c|}{ Nemátodos } & \multirow{2}{*}{\begin{tabular}{|c|} 
Céstodo \\
$\begin{array}{c}\text { Cylindrotaenia } \\
\text { americana }\end{array}$ \\
\end{tabular}} & \multirow{2}{*}{$\begin{array}{c}\text { Hirudíneo } \\
\begin{array}{c}\text { Helobdella } \\
\text { sp. }\end{array}\end{array}$} \\
\hline & $\begin{array}{c}\text { Zelleriella } \\
\text { sp }\end{array}$ & $\begin{array}{l}\text { Nyctotherus } \\
\text { cordiformis }\end{array}$ & $\begin{array}{c}\text { Hedruris } \\
\text { moniezi }\end{array}$ & $\begin{array}{l}\text { Aplectana } \\
\text { hylambatis }\end{array}$ & $\begin{array}{c}\text { Falcaustra } \\
\text { mascula }\end{array}$ & & \\
\hline Positivo & 3 & 1 & 38 & 4 & 2 & 7 & 10 \\
\hline$\%$ & 6 & 2 & 76 & 8 & 4 & 14 & 20 \\
\hline Negativo & 47 & 49 & 12 & 46 & 48 & 43 & 40 \\
\hline$\%$ & 94 & 98 & 24 & 92 & 96 & 86 & 80 \\
\hline
\end{tabular}

De los 50 B. spinolosus estudiados el $47 \%$ estaban parasitados con protozoos y/o helmintos y de los 50 Telmatobius el $47 \%$ también estaban parasitados por protozoos, helmintos y/o hirudineos. Como se puede apreciar en la tabla 1, la prevalencia parasitaria es igual en ambos huéspedes.

La relación peso-parásito de T.m. pseudojelskii se muestra en la Fig. 1

Las especies parásitas en Bufo fueron, entre los protozoos:Zeleriella sp. y Nictotherus cordiformis; entre los nemátodes: Aplectana hylambatis, Hedruris moniezi y Falcaustra mascula y el céstode Cylindrotaenia americana. Las especies parásitas que se encontraron en Telmatobius fueron las mismas que las halladas en Bufo, pero con la adición del hirudíneo Helobdella sp.. Estos datos se sumarizan en las tablas 2 y 3 . En las mismas tablas, además se señalan que el parásito de mayor frecuencia en Bufo fue el céstode $C$. americana y en Telmatobius, H. moniezi. En ambos huéspedes, los helmintos son más frecuentes que los protozoos.

\section{DISCUSION}

Pocos son los trabajos sobre parasitismo de anfibios en el Perú a pesar de la gran importancia ecológica y 


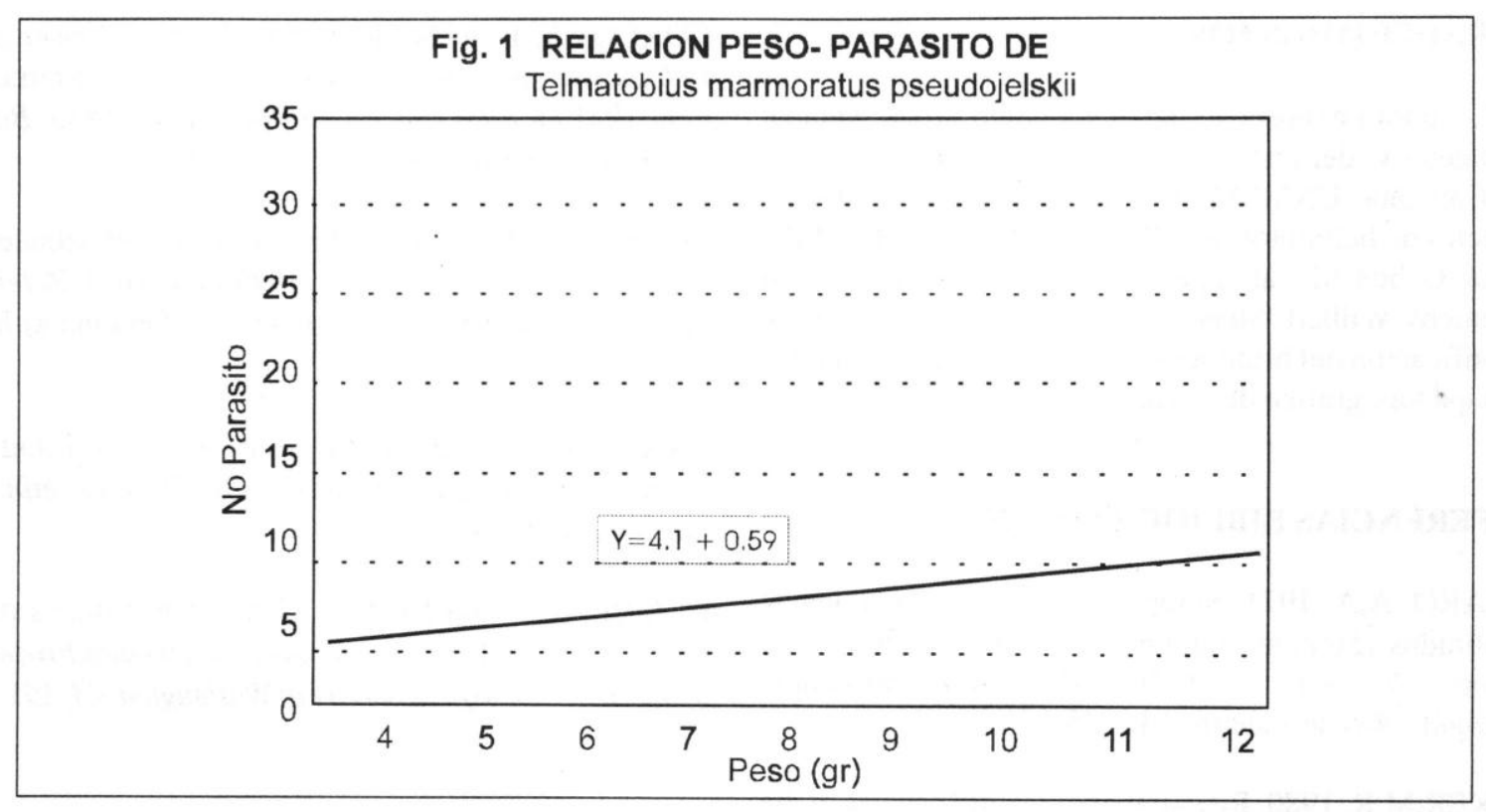

Fig.1 Relación Peso-Parásito de Telmatobius marmoratus pseudojelskii.

alimenticia de sus especies; es así que B. spinulosus constituye un importante controlador biológico y Telmatobius marmoratus pseudojelskii una fuente proteica pues se utiliza en la alimentación humana. Estas especies pueden ser criadas en granjas apropiadas, pero si no se conocen sus parasitos y enfermedades, no será posible controlar sus efectos dañinos y por tanto obtener óptimos resultados en la crianza.

Algunos parásitos son muy dañinos para los anfibios, como el nemátode Hedruris moniezi que lesiona severamente el intestino porque la hembra posee un fuerte gancho quitinoso en el extremo posterior del cuerpo con el cual se fija la mucosa intestina. Precisamente, 38 (76\%) de los Telmatobius estaban parasitados con este gusano.

Los otros helmintos hallados, aparentemente no son patógenos. Los protozoos tienen gran importancia biológica. TantoNictotherus cordiformis comoZeleriella sp. utilizan el sistema endocrino del huésped para su propia reproducción y formación de quistes infectantes; por tanto, las formas que presente el huesped pueden indicarnos el grado de maduración sexual de éste. Miranda (1978) identificó a Zeleriella peruensis en Telmatobius culeus procedente de Puno. La especie que obtuvimos de T. m. pseudojelskii podría ser la misma considerando la afinidad de los huéspedes, pero la identificación final solo será posible cuando se colecte nuevo material.

El hallazgo del Hirudíneo Helobdella sp. sobre la piel de Telmatobius tiene interés, porque aparte de ser un ectoparásito que lesiona la piel, puede ser, al mismo tiempo, transmisor de hemoparásitos.

Los helmintos que se refieren en este trabajo ya han sido anotados por otros autores en anfibios de otras localidades del Perú (Córdova y Martínez 1981; Tantaleán, 1989) y algunos de ellos en otras partes del mundo, como el céstode Cylindrotaenia americana y el nemátode Aplectana hylambatis, aunque en huéspedes diferentes (Baker,1980; Ulmer y James, 1976).

Referente a la relación peso-parásito de Bufo spinulosus se obtuvo $\mathrm{r}=0,37$ y el coeficiente de determinación $\mathrm{CD}=13,69 \%$. Esto significa que los parásitos influencian muy poco o nada sobre el peso del huesped, o sea, hay una tendencia a la independencia entre el peso y el número de parásitos. Como en este huésped observamos abundante carga parasitaria en cada individuo, es conveniente pensar que no solo debemos considerar el peso del huésped sino también el peso de los parásitos.

En cuanto a la relación talla-parásito en $B$. spinulosus se obtuvo $\mathrm{r}=0,24$ y el $\mathrm{CD}=5,76 \%$; por tanto, también existe una tendencia a la independencia entre la talla del huespéd y el número de parásitos, puesto que no siempre a mayor talla corresponde mayor número de parásitos.

En la relación peso-parásito de $T$. m. pseudojelskii $\mathrm{r}=0,75$ y el $\mathrm{CD}=56,25 \%$; por tanto, en este caso, el peso del huésped esta influenciado por el número de parásitos en 56,25\%; por consiguiente, a mayor número de parásitos es menor el peso en promedio del huésped.

Lo mismo se observa en la relación talla- parásito de este mismo huésped, donde $\mathrm{r}=0,76$ y el $\mathrm{CD}=57,76 \%$; por consiguiente, la talla del huésped es influenciada por los parásitos en $57,76 \%$; es decir, a mayor número de parásitos menor talla en promedio del huésped. 


\section{AGRADECIMIENTOS}

La autora expresa su agradecimiento al Dr. Manuel Tantaleán V. del Instituto de Medicina Tropical «Daniel A. Carrión» UNMSM. por la identificación de las especies de helmintos; a la Blgo. Flavia Muñiz P., al Dr. Oscar Ochoa M., al Ingeniero Hernán Cortés B. y al Ingeniero Wilbert Mendoza A. por el asesoramiento, identificación del hirudíneo, apoyo estadístico y facilitar el mapa topográfico de la zona respectivamente.

\section{REFERENCIAS BIBLIOGRAFICAS.}

AMARO, A.A. 1971. Sinopse das recentes especies de Opalinidos (Sarcomastigophora, Opalinata). 2da. nota: Género Zelleriella Metcalf, 1920. Atas Sociedade Biología, Rio de Janeiro, 14: 135-141.

BAKER,M.R. 1980. Revision of old world species of the genus Aplectana Railliet\& Henry, 1916 (Nematoda, Cosmocercidae). Bulletin Museum Nationale d'Histoire Naturelle, Paris 4e Ser. Sect. A, 2: 955-998.
CORDOVA, E. y MARTINEZ, E. 1981. Presencia de Falcaustra mascula (Rudolphi, 1919) Freitas \& Lent,1941 en Bufo spinulosus Weigmann, 1835. Boletín Peruano de Parasitología, 3 : 100-104.

MIRANDA, S.E. 1978. Protozoarios intestinales de Telmatobius culeus Garman, 1975 de Perú. I. Zeleriella peruensis $\mathrm{nsp}$. (Sarcomastigophora, Opalinata).Biota, 11: 192-195.

TANTALEAN, V.M. 1989. Contribución al estudio de los helmintos parásitos de anfibios del Perú.Boletín de Lima, 11: 69 - 77.

ULMER, M.J. and JAMES, H.A. 1976. Studies on the helminth fauna of Iowa. II. Cestodes of amphibians.Proceeding of the Helminthological Society of Washington, 43: 191 -200. 